\title{
LA EXALTACIÓN DE LO ROMÁNTICO AMERICANO EN LA POESÍA COLOMBIANA: ORIGEN Y DESARROLLO SEGÚN EDUARDO OSPINA
}

\author{
Jorge Chen Sham
}

\begin{abstract}
RESUMEN
La publicación en 1952 del libro El Romanticismo, estudio de sus caracteres esenciales en la poesía lírica europea y colombiana consagra al jesuita Eduardo Ospina (1891-1965) en la cima de su carrera intelectual y humanística. Su análisis del origen y evolución de la poesía colombiana supone deslindar sus rasgos estilísticos más conspicuos, para lo cual Ospina hace un balance de la intuición creadora y las ansias de trascendencia espiritual, que el Romanticismo hereda a la tradición occidental, amén de que exalta lo nacional en tanto espíritu formador de la individualidad suprasensible del poeta.

Palabras clave: Ospina-Eduardo, poesía colombiana, Romanticismo, estética, intelectuales jesuitas.
\end{abstract}

\begin{abstract}
With the publication in 1952 of his book El Romanticismo, estudio de sus caracteres esenciales en la poesía lírica europea y colombiana the Jesuit Eduardo Ospina (1891-1965) reaches the height of his intellectual and humanistic career. His analysis of the origin and evolution of Colombian poetry aims to identify its outstanding stylistic qualities, namely its balance of creative intuition coupled with a desire for spiritual transcendence, Romanticism's legacy in the West, an exaltation of the national through the creative spirit of the highly sensitive individuality of the poet.

Key words: Ospina-Eduardo, Colombian poetry, Romanticism, aesthetic, Jesuit intellectuals.
\end{abstract}

En 1952 con el título de El Romanticismo, estudio de sus caracteres esenciales en la poesía lírica europea y colombiana, el jesuita Eduardo Ospina (1891-1965) presenta en versión española lo que, en un principio, fue su tesis doctoral presentada en la Ludwig-Maximilian Universität-München por ahí de 1927, nada menos que de la mano de Karl Vossler. Como ya lo expuse en el estudio introductorio que acompaña la segunda edición en español de este

Dr. Jorge Chen Sham. Profesor de la Escuela de Filología, Lingüística y Literatura de la Universidad de Costa Rica. Miembro correspondiente de la Academia Nicaragüense de la Lengua y colaborador de la Academia Norteamericana de la Lengua Española.

Correo electrónico: jorgechsh@yahoo.com

Recepción: 04- 12- 2013

Aceptación: 24- 01- 2014 
voluminoso trabajo de Eduardo Ospina (2010: 20-21), en los años 50 se estaba difundiendo en toda Hispanoamérica las teorías estilísticas acerca del lenguaje poético y de la importancia de un análisis de las relaciones entre la intuición del escritor y su concreción literaria, de la mano de dos de los grandes discípulos de Vossler, Leo Spitzer y Dámaso Alonso. De esta manera, el desarrollo de la estilística justificaría la petición que le hacen a Ospina de publicar este trabajo suyo bajo el auspicio del Ministerio de Educación Nacional de Colombia; sin embargo, un dato biográfico es de suma importancia para comprender cómo la aparición de este voluminoso y detallado estudio vino a coronar, en la década de los 50, la ingente labor reflexiva y el magisterio docente del jesuita, el cual vino a posicionarse en la vanguardia de los estudios literarios de la época, con la publicación de su tesis doctoral aún inédita en español, para situarlo de una vez por todas en el campo de la crítica poética y de la reflexión estética. Este movimiento de consagración culmina en 1957, cuando pronunció, el 9 de setiembre, su discurso de incorporación a la Academia Colombiana de la Lengua con el trabajo "El motivo más artístico del Evangelio" (Ospina 2008). Sus méritos intelectuales y su trabajo crítico se reconocen de esta manera.

En mi introducción a El Romanticismo, estudio de sus caracteres esenciales en la poesía lírica europea y colombiana, expuse las ideas teórico-estéticas que sustentan su concepción del lenguaje y del arte, apegadas a la noción de la expresividad de la forma poética, la valoración de lo musical en la experiencia de las emociones, la revelación del espíritu ideal que informa al poeta, para terminar subrayando esa radical visión del pensamiento religioso que una parte del Romanticismo expone en su acercamiento a la divinidad y al papel del arte como búsqueda que conduzca a la iluminación y a la revelación de la unidad reintegrativa de la humanidad (Chen 2010: 35-36). Subrayaba, por otra parte, que para Ospina la "forma interna" del Romanticismo posee una constante histórica que se manifiesta en las literaturas nacionales. Es expresión de un invariante que se desarrolla en cada país y en cada época, dependiendo de esos accidentes geográficos e históricos que caracterizan su gestación.

Retomando la propuesta de Vítor Manuel Aguiar e Silva (1979) en materia de periodización, Francisco Gutiérrez Carbajo (2003) señala que dos definiciones han dominado las orientaciones de los que repertorian y plantean un estudio sistemático y metódico de los fenómenos literarios desde el punto de vista de la historia literaria: a) la actitud nominalista, "que considera el concepto de etapa o período como una simple etiqueta intercambiable, desprovista de todo contenido real (Gutiérrez 2003: 34) y, por lo tanto, se decanta por una definición histórica y evolutiva de los movimientos literarios; y b) la actitud metafísica, "según la cual las épocas o períodos son entidades transhistóricas" (Gutiérrez 2003: 34), a causa de lo cual se anula el hecho de que las obras literarias se enmarquen y se ubiquen dentro de una historicidad. Así las cosas, ya desde el Capítulo I de la Primera Sección "Caracterización de la lírica en cuatro románticas europeas, por método histórico-crítico", Eduardo Ospina nos plantea una concepción de Romanticismo que desemboca en una definición metafísica en materia de periodización, por cuanto se plantea como:

a. Escuela filosófica y no literaria, cuyos rasgos no son el productor o la determinante de la "forma externa" retórica o del uso del lenguaje en sí mismo, sino que parte de la "forma interna", producto de una concepción vital y conceptual que tenga el poeta. De esta manera, la literatura es "expresión de la propia vida" en esa interpenetración entre la obra y las motivaciones del poeta. Es decir, el Romanticismo es ante todo una concepción vital. 
b. A la luz de lo anterior, si esa "forma interna" permanece y traspasa un momento histórico, es porque los poetas y artistas seguirían aceptándolos como necesarios e indispensables, gracias a lo cual permanecería invariable y transhistórica, en donde "lo afectivo y lo conceptual" no se pueden divorciar porque son una unidad indisoluble que proviene de la afectividad profunda y son el producto de "las reacciones inmediatas a los estímulos vitales" (Dámaso Alonso 1981: 194). Ese es el objetivo final, cuando Ospina se lo propone de la siguiente manera: "Si el arte posterior hasta nuestros días está envuelto en los mismos rasgos fundamentales que el primer romanticismo, eso significaría que de mis principios se deduce que es romántico el arte moderno y que el romanticismo vive todavía" (2010: 40). Estos "caracteres esenciales", como anuncia en el título de su libro, son universales.

De esta manera, el estilo es la expresión meditada y exaltada que el poeta intuye por medio de la creación y Ospina descubre en el estilo romántico unas raíces filosóficas que la índole psicológica/vital del artista transvasa a su arte creativo, de manera que los "caracteres esenciales" del Romanticismo están subordinados a una visión metafísica que ordena el programa analítico y descriptivo del jesuita colombiano. Él parte de una concepción dialéctica, establecida por lo demás a priori, para describir los fenómenos y los pone dentro de unas categorías que hacen de las obras literarias expresión sentimental y espiritual de su creador; por ello su presencia y su vigencia en otras regiones como Colombia es innegable en esa sintonía de almas gemelas y en una comunión espiritual, pues como indica su biógrafo, se trata de una transformación cuya eficacia es perceptible en el mundo moderno (Miranda Ribadaneira 1980: 106).

El Romanticismo sería, para él, una corriente y un ideal en el arte; las circunstancias y las vicisitudes temporales de las obras permiten descubrir que, por encima de aquellas, guardan inalterables y en su radicalidad más profunda la esencia de su creador (Aguiar e Silva 1979: 268). De esta manera, Ospina vuelve sobre el pensamiento filosófico de la estética romántica más radical, la cual depura el ideal de la forma en el clasicismo greco-latino frente al desarrollo del símbolo absoluto de eminente expresividad moderna, tal y como lo expone Hegel en sus Lecciones sobre estética (1834), en esa irradiación del espíritu hacia la unidad de la forma; aseguraba el filósofo alemán lo siguiente: "El espíritu tiene por esencia la conformidad consigo mismo, la unidad de su idea y de su realización. No puede, por tanto, encontrar la realidad que le corresponde sino en el mundo propio, el mundo espiritual o interior del alma" (Hegel 2003: 210, las cursivas son del texto) ${ }^{1}$. Hegel terminará por dar cuerpo a ese debate que, en estética, pondera el arte moderno del Romanticismo frente al arte clásico, a partir de esta correspondencia entre espíritu y forma simbólica, por lo que hace oscilar el debate estético entre dos polos: clasicismo y romanticismo; así, este último se afirma y se construye como estética revolucionaria sobre el primero. Su corolario será pensar que en la persistencia/oscilación entre un espíritu apolíneo y un espíritu dionisíaco se va alineando las obras artísticas de un periodo histórico determinado ${ }^{2}$.

Por ello, el Romanticismo es, para Eduardo Ospina, una constante histórica que, en su paso y en viaje por tierras americanas, guarda intacto la fuerza y la conciencia metafísica que tanto preocupaba en tierras europeas. Para ello, el crítico nos propone un estudio comparativo de interrelaciones que buscan ver "los invariantes de la imaginación literaria, el alfabeto transhistórico en el que se fijaría universalmente la lengua de la creación estética humana" (González de Avila 2001: 11, la cursiva es del autor), de lo cual se sigue que su 
comparación se basa en la puesta en relación de un número de propiedades. El repaso que hace Ospina a las poesías inglesa, alemana, francesa y española del Romanticismo supone una operación hermenéutica en la que encontramos los mismos problemas vitales y las respuestas metafísicas a estos problemas que identifican y hacen comulgar a los creadores y los hacen almas gemelas, al tiempo que ello supone también que sus obras poéticas responden a la misma función estética, de dar una respuesta en su cosmovisión filosófica a esas inquietudes que sacuden el espíritu humano. La línea que escoge Ospina es segura y se enmarca en toda una tradición de estudios comparados sobre el Romanticismo, cuya vigencia y correspondencia es aún válida en el ámbito de la crítica literaria ${ }^{3}$.

Y todo este recorrido culmina con el estudio del romanticismo en la poesía colombiana, para lo cual remite a lo que la crítica decimonónica privilegia, es decir, la biografía del creador y la individualidad del artista, pues: a) los rasgos morales-psicológicos o culturales del espíritu de este creador iluminan la obra, y b) la original personalidad del artista solo se explica en ciertas disposiciones que están dadas en la raza, el medio y la historia. Por lo tanto, el estudio metódico de la obra artística se subordinaba "a exigencias de rigor y objetividad" (Aguiar e Silva 1979: 361), como lo hace el positivismo científico y que la estilística asume en el arte. Gracias a esta "comunión espiritual", que encuentra Ospina entre el poeta y el espíritu absoluto primero, y luego entre la obra y la personalidad creadora, Ospina no solo puede devolverle al subjetivismo (léase intuición y sensibilidad) su lugar, sino también vincular la obra de arte con "características espirituales y de corazón comunes a los hombres de una raza, de un siglo o de un país" (Hipólito Taine, citado por Aguiar e Silva 1979: 348). Y eso es lo que realiza Ospina, cuando al principio de la "Segunda Sección" de su voluminoso ensayo, la dedicada propiamente a la poesía colombiana; se hace una serie de preguntas a las que responderá a continuación:

\footnotetext{
¿Se debe a que la raza, adaptada por una larga aclimatación, se ha hecho más sensible para vibrar sintónicamente con la maravillosa belleza del medio ambiente? ¿Se debe a la configuración topográfica del territorio colombiano, que, más montañoso y variado que el de ninguna otra nación americana, presenta todos los climas, desde las altas mesetas o sabanas primaverales hasta los ardientes valles y costas? ¿Se debe al mismo retiramiento en que la nación permaneció hasta hace poco enfrente al vertiginoso progreso europeo y le permitió conservar una vida aristocrática en su sencillez, muy propensa a la lectura, al arte y a la poesía? (2010: 206-207, la cursiva es del autor)
}

Según Ospina, la propensión de la raza hispana hacia las más altas pasiones y la orografía del medio son ese clima espiritual que nutre el arte; en este sentido, las variaciones topográficas y las modulaciones del clima, aunado a esa mentalidad de encerramiento y de retiro que ha sufrido el espíritu colombino, el que se ha forjado en los valles cercados por las montañas de los Andes, hacen que la nacionalidad colombiana se preste para que el romanticismo se arraigue en estas tierras, en una sintonía que Ospina ve en dos coordenadas muy específicas:

a. La correspondencia "entre las alternativas de esa vida [se refiere a la psicológica] y las alternativas de la poesía" (2010: 211). Es decir, plantea Ospina la indisoluble relación entre las elecciones estilísticas del poeta y sus estados emocionales, en lo que será una constante en las propuestas estilísticas: ¿qué experimentó el poeta al crear tal obra? Ospina busca, pues, la correspondencia o la equivalencia entre las motivaciones y sus circunstancias de creación. 
b. La individualidad del artista y su "concepción suprasensible de la vida" (2010: 211, la cursiva es del autor). Este nivel permite ver la jerarquía axiológica en la que la ética se erige en el principio rector del arte, porque se cuestiona, a pesar de lo que digan las vulgatas de la estética del Romanticismo que ponderan el arte por el arte como privilegio ostensible de esta estética, se cuestiona, decíamos, por la posición y la respuesta que el poeta proporciona a la realidad. La pregunta sería, obviamente, ¿a cuál concepción de la vida y del cosmos responde? A lo que responde Ospina subrayando el privilegio de "los problemas metafísicos relacionadas con la personalidad [del artista]" (2010: 213), lo cual conduce a valorar la dicotomía de la superficialidad/la profundidad a la que aspira la obra de arte en tanto "proyección del mundo psicológico del poeta sobre el mundo visible" (2010: 213, las cursivas son del texto). La profundidad de la obra, premisa sobre la que descansa toda la concepción de obra de arte en el Romanticismo (Aguiar e Silva 1979: 68) ${ }^{4}$, hace que la apariencia del mundo visible conduzca a un "mundo superior" (2010: 213, las cursivas son del texto) en el que el poeta aspira hacia el infinito, a la trascendencia que solamente puede un alma sensible llegar a acercarse por medio del hecho poético.

El corolario de este estatuto profundo o simbólico de la poesía será la oposición entre el mundo externo y mundo superior, con esa necesidad propia de la literatura romántica a "divulgar los secretos de la interioridad humana, [...] los más íntimos repliegues del alma y del cuerpo" (Aguiar e Silva 1979: 322). La imaginación onírica, la meditativa reflexión que se recobra en el espacio de la noche o del alba configuran esas vías privilegiadas para que la instrospección sensible conduzca tanto "a un conocimiento más profundo de la psicología individual” (Béguin 1993: 45), como a las intuiciones místicas de la naturaleza.

Así como ser privilegiado y con vocación intimista, también para Ospina el poeta posee una "visión ultrasensible" (2010: 215) que descubrirá primeramente en "la parte más externa y material del mundo" (2010: 216), es decir, en la naturaleza que le rodea al ser humano o lo que él llama simplemento en el título capitular como "El mundo externo" (2010: 215). Para ello, en esa correlación medio natural y exaltación del espíritu colectivo, Ospina destaca, en efecto, el privilegio de la selva, el agua, el viento, los astros, los árboles en el repertorio romántico colombiano; nombres como José Eustasio Rivera o Rafael Pombo surgen en primera línea y no son casuales cuando se trata de ver la selva en sus "misteriosas inspiraciones y enseñanzas profundas" (2010: 217). También elementos terrestres y acuáticos se privilegian para terminar en lo que Michel Collot denomina como propio del "voilement-dévoilement de l'Être" (1989: 35) de la experiencia del horizonte; cuando el ser humano mira hacia este, "il signifie à la fois la circonscription d'un champ objectal par l'activité perceptive du sujet, et son débordement par le fond infini du Monde" (Collot 1989: 38).

Por eso, el crepúsculo y sus cambios de luz, la luna y las estrellas cobran un privilegio especial. Mención especial posee R. Núñez con su poema "Véspero" cuando "la luz empieza a languidecer" (2010: 226) o Diego Fallon con "La luna", para quien expresa Ospina toda su admiración: "Confieso sinceramente que en ninguna literatura he encontrado una descripción más bella de la aparición y ascenso de la luna" (2010: 229). En cuanto a esa relación de lo natural con el mundo de las aves, no nos extrañe que Ospina les dedique su espacio, porque representan el vuelo ascensional y la aspiración humana a la imaginación ensoñadora. A Guillermo Valencia lo pondera por su poema "Cigüeñas blancas" (2010: 233-234), mientras 
que A. Martínez Mutis es celebrado por "La epopeya del cóndor" (2010: 237-238), para terminar su atención en materia del espacio autóctono y nacional con el tema de "La Patria", la cual define "primariamente al propio territorio donde se contienen todos los elementos que integran el concepto: éste dice región, raza, familia, costumbres, culto, historia y todos los intereses sociales inmediatos" (2010: 238).

En materia de esta celebración del sentimiento patriótico, señala Ospina en primer lugar a Rafael Núñez, quien compuso la letra del Himno Nacional de Colombia y destaca sobre todo su estribillo que dice: “¡Oh gloria inmarcesible!/ ¡Oh júbilo inmortal!/ En surcos de colores/ el bien germina ya" (2010: 239); su comentario es lúcido y atinado sobre este himno, cuando relaciona las luchas a favor de la independencia con la exaltación del yo poético: "despliega líricamente sobre el cuadro del patrio suelo la epopeya que produjo la autonomía nacional" (2010: 239). Entramos, pues, en el sentimiento patriótico que como valor civilista encuentra ejemplos Ospina en Rafael Pombo con su poema "Todo por mi patria" o en R. Nieto con su "Himno a la bandera", en donde el emblemático símbolo pasa a ser equivalencia antonomásica de la "nación" colombiana.

Luego, en el capítulo más enjundioso, el tercero, Ospina lo dedica a desentrañar su tesis central que muy bien explica en su título: "La proyección del alma sobre el mundo visible" (2010: 261), es decir, privilegia "la percepción interior" y sus principios estéticos. Por supuesto, su primer comentario lo conduce a reconocer la excepcionalidad del poeta en tanto ser con un privilegio de conciencia y de exaltación interior, pues en estos genios "la espiritualidad de la vida interior y la reflexión subjetiva han llegado a tan alto grado, que -por una paradoja psíquica- se actúan sin reflexión y se funden a los actos espontáneos sensibles y aun sensitivos" (2010: 262). La superioridad del creador es indiscutible para Ospina, quien se silencia para abordar la personalidad agudizada y ultrasensible de algunos creadores románticos que para él serían expresión nada más de "vanas fantasmagorías" (2010: 262).

Volviendo sobre lo propuesto por Ospina, llama poderosamente la atención desde un punto de vista hermenéutico el hecho de que ahora sí analiza los poemas y que no haga un simple repertorio de citaciones como sucede principalmente en el capítulo anterior. El mismo aclaraba al inicio de su exposición que de lo que se trata aquí es de "la comprensión de ese principio" (2010: 261) gracias al cual el poeta transvasa su verdad íntima en el arte y que se capta en la forma "interior" del arte, en el plano de la creación. El primer ejemplo ofrecido por Ospina, referente a la naturaleza, nos permitirá un acercamento inicial a esta problemática. Se refiere Ospina al poema "El Mar Muerto" de R. Núñez, en donde encuentra dos partes dentro de un modelo compositivo analítico ${ }^{6}$, en donde el objeto externo, el Mar Muerto y la destrucción de Sodoma y Gomorra, se aplica, afirma Ospina, a una realidad interior; las dos partes del poema, afirma él, "no están dislocadas; brotan al tiempo, fundidas íntimamente en el alma de un poeta reflexivo y sombrío, que se concentraba por temporadas en una meditación lóbrega sobre el misterio de la vida y el misterio de sí mismo" (2010: 263). A estas interrelaciones entre el plano expresivo y la intuición del poeta es lo que se denomina como motivación del signo poético, con el fin de que esa "visión de mundo" sea parte integrativa del "acto de construcción de base estética" (Amado Alonso 1969: 83) según expone la teoría estilística.

La preeminencia de esta visión interior del poeta que se manifiesta bajo esa intuición de que reproduce el acto creador es de lo que tratará en el primer apartado del "Capítulo IV". Ospina parte de esa afirmación propia que enuncia el Romanticismo ante la esencia del arte, que escapa a lo cognoscible y palpable y, más bien, nos acerca hacia lo inefable y la 
trascendencia del símbolo. Por ello, en esa vía que del cosmos conduce hacia la interioridad del ser para después desplegarse hacia la exaltación sobrecogedora, Ospina dirá que "éste [se refiere al impulso creador romántico] empuja por sí mismo hacia esa visión interior que no tiene límites" (2010: 287). Las fronteras se neutralizan y el acto estético conduce hacia una meditación que está en las bases mismas del valor profundo del símbolo romántico; no nos extrañe, entonces, las razones por las cuales Eduardo Ospina se detendrá con vehemencia en el "abismo interior" y lo que él llama metafóricamente como el "Cielo interior" (2010: 292); en estas dos categorías encontrará respectivamente el dolor acendrado que "sondea el abismo de la existencia adolorida" (2010: 290) y la lámpara interior de la reflexión, en esa transición del sueño al amanecer renovador y seguro a causa de su luminosidad. Noche y alba se convertirán en los dos tópicos que mueven a la contemplación; para el primero reproduce el intenso poema de reminiscencias sanjuanianas "Las dos noches" de Gregorio Gutiérrez González; para el segundo selecciona de Joaquín González Camargo lo que en el texto es un viaje significativo a la semilla para luego impulsarse hacia las cumbres luminosas en "Viaje de la luz".

No podría faltar tampoco en esta caracterización del símbolo inefable, que es la existencia humana, su dualismo fundador desde el punto de vista platónico y cristiano; así lo expone con gran contundencia en el principio del segundo apartado del "Capítulo IV":

\footnotetext{
Era imposible que en esa mirada escudriñadora y sentida que el poeta romántico dirige a su mundo interior no tuviera muchas veces la íntima y doliente sensación de la duplicidad que se unifica sin identificarse en la esencia de la personalidad humana: cuerpo y alma, materia y espíritu. Y es ésta una sensación casi siempre dolorosa, porque se experimenta precisamente en los que la parte celeste quiere remontarse hacia la altura y siente el peso de la parte de tierra en que está sumergida. (2010: 295-297) ${ }^{7}$
}

Observemos en primer lugar la "carga" que pesa sobre las oposiciones encontradas: cuerpo/alma, materia/espíritu; el desbalance es inmediato para lo que nuestra tradición occidental hace de la materia la prisión del "alma". Así, el dualismo humano funciona como una realidad "dolorosa", dice Ospina, para el poeta sensible y en búsqueda de trascendencia, que la poesía le ofrece en cuanto experiencia de reintegración. Si el cuerpo pesa por ser lastre, su primera consecuencia es que sea "prisión", tal y como la percibe Rafael Pombo en su doliente "Vaguedad", para que después, con su contraste u oposición, se vean los dos polos en que gravita este dualismo y Ospina se detenga en la ligereza de las cosas aspirando a la levedad del alma, como expone el mismo Pombo en otro poema, "En la cama". Por su parte, la meditación del nocturno hace posible esta "distensión" (2010: 299) del conflicto íntimo del ser humano que encuentra Ospina en el poema "Némesis I" de C. A. Torres. Esta pesada carga es la que coarta toda empresa vital y, por ello, el amor y el dolor se tiñen de estos matices. Para este primer sentimiento acude a J. E. Caro y a su amada, que este personificaba en "La hurí", para confrontar su presencia en una figura que se escapa y no puede aprisionar el yo lírico; se trata de esa imagen propia de la elevación y de la intensidad etérea, cuando Rafael Maya, en "Tú", la comparaba con "[a]ire ligero/ cernido entre las flores y los nidos" (2010: 304). Para el intenso sentimiento del dolor, cuyas quejas "repercuten en el infinito" (2010: 307) y son el germen de toda aspiración humana hacia la trascendencia, Ospina presenta el acuciante poema de E. W. Fernández; su título, "El dolor", ya ponía en evidencia la temporalidad humana, mientras el tema mitológico del "Prometeo" de R. Núñez le permite destacar "la conciencia de la grandeza del dolor [humano]" (2010: 313).

Faltaría abordar la función que poseen la trascendencia y el absoluto en el programa interpretativo de Eduardo Ospina; le dedica todo un capítulo, el Quinto, a lo que él explicita como "El mundo superior", pues encuentra en el apogeo del Romanticismo europeo las huellas 
penetrantes de un pensamiento religioso que, sobre todo, el materialismo propagado por la filosofía positivista decimonónica plantea a la hora de separar definitivamente filosofía y religión. De esta manera, en sus planteamientos Ospina encuentra que ni la ciencia ni la filosofía del siglo XX "logran dar solución aquietadora para las grandes cuestiones humanas sobre el destino del hombre" (2010: 332), mientras que en la poesía colombiana sí que se manifiesta esta preocupación por lo superior y el infinito. En primer lugar, el jesuita se detiene en la dimensión de la "objetividad superior" (2010: 333), cuyo instrumento de conocimiento, asegura él, es la fe, y la vincula con la esperanza, por cuanto "la clave del enigma de la vida, del amor, del dolor, es la esperanza" (2010: 334); varios poemas de La esfera conquistada, de A. Martínez Mutis, le sirven para ejemplificar estas insondables ansias de infinitud y divinidad, en unos poemas que revelan el lado visionario y metafísico de la experiencia que buscaba Martínez Mutis por medio de la poesía. Después analiza la repercusión del cristianismo ${ }^{8}$ en la poesía colombiana del Romanticismo; correspondencia que percibe gracias a esa equivalencia que encuentra Ospina "en la estructura psicológica del romántico y el infinito cristiano" (2010: 336), lo cual desemboca en la irradiación de la divinidad en la naturaleza creada y en la pequeñez del ser humano, tal y como observa en "Indiferencia" de Rafael Pombo cuando el yo poético confiesa su impotencia ante ese cosmos que se le abre ante la inmensidad de sus ojos (2010: 339). Termina esta sección repasando la presencia de las figuras de Cristo o de la Virgen María en algunos poetas.

A la luz de lo anterior, el centro neurálgico de su exposición en este capítulo dedicado al problema de lo divino es el destino del ser humano, lo cual es el motor de toda comprensión de la trascendencia en el misterio de la vida y de la muerte; así lo expone con gran acierto en un afán de clasificación:

\footnotetext{
Una de las ideas que han producido en los románticos más sinceros [con] "estremecimientos metafísicos" es la idea o la preocupación del destino humano, que, en sustancia viene a ser la preocupación por el infinito “¿De dónde? ¿Adónde?”. Esto es aún más de notar en los poetas incrédulos que en los creyentes. Los creyentes que tienen en su fe una solución exacta, terminante, satisfactoria de esas angustiosas preguntas, miran al porvenir con paz y esperanza. Los incrédulos miran ese más allá inexplorado y enigmático en una inquietud dolorosa y sombría. (2010: 354, las cursivas son del texto)
}

La explicación ulterior de la vida se repertoria, según Ospina, en la poesía colombina con unos matices de "intensidad radical" (2010: 354), porque el "más allá" produce unas tensiones en los dos bandos antes mencionados; la respuesta metafísica se impone en esa meditación que conduce a algunos a un "sentimiento íntimo y melancólico" (2010: 356) como en E. W. Fernández y su implacable poema "El dolor". Otras veces adquiere, asegura él, un "tinte melancólico" (2010: 357) cuando se interroga esa "hora secreta" a la que el ser humano está convocado y se manifiesta en el poema II de La esfera conquistada, de A. Martínez Mutis. Lo anterior nos conduce inexorablemente a la problemática de la muerte y de las dudas existenciales, por ejemplo, ante la "angustia extraviada" (2010: 359) de C. A. Torres y la voz quejumbrosa del yo poético en el poema I de Némesis. Merece otra vez la atención de Ospina el poeta Rafael Pombo, a quien encuentra hermanado al romanticismo más auténtico en su búsqueda de inmensidad y ansiedad metafísica, cuando en el soneto "De la noche" expresaba su palabra de "melancólica resignación y de tranquila esperanza" (2010: 363). Porque en estas circunstancias, el yo poético se eleva, necesariamente, hacia una tabla de salvación que puede encontrar hacia las alturas de las estrellas, según se explayaba J. M. Rivas Groot en el largo poema "Las constelaciones", soliloquio interminable hacia "la irremediable nostalgia de lo eterno" (2010: 366). O lo puede hacer también, aunque parezca contradicción, por medio de lo material y finito, es decir, cuando 
se descubre en las cosas "los fulgores suprasensibles" (2010: 369); varios caminos se imponen, ya sea la naturaleza y el cosmos que despliegan su maravilla a través de las zonas temporales del día, en especial, el nocturno o el crepúsculo, ya sea por medio del arte, del amor o del dolor. Para todas estas categorías Ospina pone entrañables y cimeros ejemplos de la poesía de Casas, Fallon,Valencia, R. Núñez y Pombo.

En conclusión, en 1927 ya se propone Eduardo Ospina un estudio de una parcela de una literatura nacional (la poesía colombina) y la estudia en sus correspondencias con una de las grandes corrientes de la estética occidental, el Romanticismo. El gesto no puede pasar desapercibido desde el punto de vista historiográfico, porque en un momento en que triunfan las vanguardias en cuanto concepción estética y en narrativa se desarrollan las corrientes de la novela regionalista, la crítica hispanoamericana apenas comenzaba su camino de emancipación y se dibujaba en el caso colombiano, esa relación y ese ligamen con la literatura mundial, muy propio de los estudios literarios latinoamericanos hasta el último tercio del siglo XX. El primer gesto es este que realiza Eduardo Ospina, concebirla desde una dinámica de un territorio nacional como lo hace el jesuita y, de este modo, enmarcarla dentro de las grandes corrientes de la literatura universal, con el fin de que a la altura de los años 60, cuando emerge el Boom latinoamericano como realidad palmaria, empiece esa emancipación crítica y teórica que permitirá la reivindicación de lo producido en tierras americanas.

\section{Notas}

1. Las coincidencias entre Ospina y Hegel son más significativas de lo que pensamos. Su contraposición entre la forma clásica y la romántica, la depuración del ideal absoluto de la forma, la función de la religión en el arte acercan el pensamiento del colombiano al filosófo alemán en este libro culmen de la estética romántica. Lamentablemente el espacio limitado de este trabajo no permite desarrollarlos en forma sistemática y comparativa.

2. Aguiar e Silva expone las consecuencias de este modelo oposicional entre lo clásico/lo romántico en un dilema de alcances míticos: "En todas las épocas y en todos los lugares, el hombre y el artista, la vida y la cultura, reviven el dilema fáustico: o la opción de la ascesis, del rigor y de la disciplina del espíritu, o la opción de la exuberancia vital y del goce apasionado delmundo, vendiendo el alma a Mefistóteles —es decir, en términos no míticos, la opción del clasicismo o la del [romanticisco]" (1979: 268). Además, recordemos que es también la propuesta de Friedrich Nietzche en El nacimiento de la tragedia.

3. Aunque habría que agregar que no lo es desde un punto de vista epistemológico, pues la teoría literaria contemporánea ha cuestionado desde hace mucho este tipo de planteamientos metafísicos en lo que se refiere a la evolución literaria y al valor estético.

4. Veamos lo que afirma Aguiar e Silva al respecto: "En la estética romántica, la poesía es concebida como la única vía de conocimiento de la realidad profunda del ser, pues el universo aparece poblado de cosas y de formas que, aparentemente desprovistas de significado, constituyen la presencia simbólica de una realidad misteriosa e invisible" (1979: 68).

5. La palabra es mía y no debe atribuírsele a Ospina, quien creeemos no la utilizaría por sus connotaciones religiosas en el contexto de la estética romántica, pues el genio es un semidios o un demiurgo creador.

6. Tanto por su esquema compositivo (unidad temática, extensión y relación de sus dos partes) este poema se ajusta a una organización dicotómica siguiendo la termonología de López-Casanova (1994: 36-37); la primera unidad retoma el intertexto bíblico de la destrucción de los impíos en Sodoma y Gomorra (16 versos), mientras que en su segunda parte (9 versos) se hace la comparación degradativa en forma de lamentatio en el que se expone el tópico de las vanas ilusiones.

7. No se trata de un error de paginación, en la edición que manejamos toda la página 296 reproduce una nota de pie en la que Ospina pone ejemplos en las poesías alemanas y francesas, por lo que su texto continúa en la página 297. 
8. Para estos aspectos remito al lector a los dos libros de Abrams, citados en la bibliografía y a mi introducción al texto de Ospina. La extensión de este artículo no me permite la confrontación extensa y reflexiva del débito de Ospina a las ideas románticas acerca de la divinidad, la concepción del arte, la reflexión de los medios expresivos, que sí hago en mi trabajo introductorio. El segundo libro de Abrams nos acerca, a este respecto, a un diálogo con esa trascendencia que el Romanticismo, inglés y francés sobre todo, quieren ver en el proyecto del cosmos y del ser humano; remito a este libro para quien desee profundizar.

\section{Bibliografía}

Abrams, M. H. 1962. El espejo y la lámpara: Teoría romántica y tradición clásica acerca del hecho literario. Buenos Aires: Editorial Nova.

1992. El Romanticismo: Tradición y revolución. Madrid: Editorial Visor.

Aguiar e Silva, V. M. 1979. Teoría de la literatura. (3ª reimpresión). Madrid: Editorial Gredos. Alonso, A. 1969. Materia y forma en poesía. (3ª edición). Madrid: Editorial Gredos.

Alonso, D. 1981. Poesía española: Ensayo de métodos y límites estilísticos. (3a . reimpresión). Madrid: Editorial Gredos.

Béguin, A. 1993. El alma romántica y el sueño: Ensayo sobre el romanticismo alemán y la poesía francesa. (2 $2^{\mathrm{a}}$. reimpresión). México, D. F.: Fondo de Cultura Económica.

Chen Sham, J. 2010. "La estética del Romanticismo y su depuración filosófica en Eduardo Ospina (Una introducción). Eduardo Ospina”. El Romanticismo: Estudio de sus caracteres esenciales en la poesía lírica europea y colombiana. San José: Editorial Promesa.

Collot, M. 1989. La poésie moderne et la structure d'horizon. París: Presses Universitaires de France.

González de Ávila, M. 2001. Sobre literatura y comparación. Letras de Deusto. 31 (92): 9-28.

Gutiérrez Carbajo, F. 2003. Movimientos y Épocas literarias. Madrid: Publicaciones de la UNED.

Hegel, G. W. F. 2003. Lecciones sobre la estética. Madrid: Mestas Ediciones.

López-Casanova, A. 1994. El texto poético: Teoría y metodología. Salamanca: Ediciones Colegio de España.

Miranda Ribadeneira, F. 1980. Eduardo Ospina, S.J.: Humanista colombiano 1891-1965. Bogotá: Editorial Kelly.

Ospina, E. 2008. "El motivo más artístico del Evangelio". La belleza objetiva. San José: Editorial Promesa.

2010. El Romanticismo: Estudio de sus caracteres esenciales en la poesía lírica europea y colombiana. San José: Editorial Promesa. 